\title{
Transatlantica
}

Revue d'études américaines. American Studies Journal

Elliot Liebow, Tally's Corner : Les Noirs du coin de la rue, traduction et préface de Célia Bense Ferreira Alves, Rennes, Presses Universitaires de Rennes, Collection « Le sens social », 2010

\section{Catherine Pouzoulet}

\section{OpenEdition}

\section{Journals}

Édition électronique

URL : http://journals.openedition.org/transatlantica/5238

DOI : 10.4000/transatlantica.5238

ISSN : 1765-2766

Éditeur

AFEA

Référence électronique

Catherine Pouzoulet, «Elliot Liebow, Tally's Corner : Les Noirs du coin de la rue, traduction et préface de Célia Bense Ferreira Alves, Rennes, Presses Universitaires de Rennes, Collection « Le sens social », 2010 », Transatlantica [En ligne], 1 | 2011, mis en ligne le 30 octobre 2011, consulté le 29 avril 2021. URL : http://journals.openedition.org/transatlantica/5238 ; DOI : https://doi.org/10.4000/ transatlantica. 5238

Ce document a été généré automatiquement le 29 avril 2021

\section{cc) () $९$}

Transatlantica - Revue d'études américaines est mis à disposition selon les termes de la licence Creative Commons Attribution - Pas d'Utilisation Commerciale - Pas de Modification 4.0 International. 


\title{
Elliot Liebow, Tally's Corner : Les Noirs du coin de la rue, traduction et préface de Célia Bense Ferreira Alves, Rennes, Presses Universitaires de Rennes, Collection « Le sens social », 2010
}

\author{
Catherine Pouzoulet
}

1 Initialement publiée en 1967, la monographie d'Elliot Liebow, Tally's Corner: A Study of Negro Streetcorner Men, s'est imposée comme un classique de la sociologie urbaine américaine, qui, dans cette nouvelle parution aux Presses Universitaires de Rennes, est maintenant accessible au public français dans la traduction de Célia Bense Ferreira Alves, sociologue à l'Université Paris VIII. Enrichi d'une longue préface de la traductrice et accompagné d'une introduction et d'une annexe méthodologique de l'auteur, ce texte constitue un excellent document de travail sur le contexte politique des années soixante et sur la méthodologie d'une enquête sociologique.

2 Le travail de terrain qu'entreprend en effet Elliot Liebow en 1962 dans le cadre de la préparation d'une thèse de doctorat en anthropologie de la Catholic University of America s'inscrit dans un projet plus large, une enquête sur l'éducation des enfants dans les familles à bas revenus du District de Columbia conduite par le Health and Welfare Council grâce à une subvention du National Institute of Mental Health. Créé en 1949, cet organisme fédéral avait reçu en effet de généreux financements pour mener «au plan national une analyse et une réévaluation objectives et complètes des problèmes économiques et humains liés à la santé mentale » (13) à la suite de l'adoption en 1955 d'une loi sur l'étude de la santé mentale (Mental Health Study Act). De fait, l'Institut met en place au début des années soixante des centres de recherche sur «la schizophrénie, la santé mentale des enfants et des familles, le suicide, la délinquance, les questions de santé 
mentale des minorités et les problèmes urbains" (13). Cette volonté affirmée de résoudre les problèmes sociaux en les abordant de manière scientifique allait devenir une démarche caractéristique du "libéralisme» de l'ère Kennedy-Johnson. Tally's Corner porte ainsi la marque d'une confiance dans le "social engineering»: cette capacité réformatrice du gouvernement fédéral souleva de grands espoirs avant d'être démentie par les émeutes urbaines de la fin des années soixante et par la radicalisation des militants noirs. Elle fut ensuite désavouée par les nouveaux conservateurs qui contestaient le principe même de l'activisme gouvernemental en matière sociale.

Elliot Liebow s'est ainsi trouvé recruté pour conduire, au sein d'une enquête sur les conditions de vie des enfants de milieux défavorisés, un travail de terrain novateur autour des « hommes de la rue » d'un quartier pauvre de Washington, D.C. (le terme de ghetto ne sera jamais employé), qui jusque-là n'avaient fait, à la différence des femmes et des enfants concernés par les mesures d'aide sociale (en particulier le programme AFDC, Aid to Families with Dependent Children), l'objet d'aucune étude sérieuse. À un moment donc où les pouvoirs publics entreprenaient de mobiliser ressources publiques et privées «dans un effort concerté d'éradiquer du pays la délinquance et la dépendance » (29), il avait paru urgent aux chercheurs de corriger la myopie des études antérieures qui tendaient à négliger l'homme noir de classe populaire, parce qu'il était moins visible et accessible, et que de surcroît, il était considéré « comme une personne ne nécessitant pas ou ne méritant pas l'aide de la société » (30). On avait de ce fait eu trop tendance jusque-là à donner une représentation des familles noires vivant dans la pauvreté comme d'un «monde peuplé de femmes et d'enfants» (30). Mais la guerre contre la pauvreté déclarée par l'administration Johnson s'accommodait mal de données aussi lacunaires sur les classes populaires. Et il n'était pas possible de continuer à définir l'homme adulte comme "absent ", ou à le dépeindre comme "une sorte de figure fantomatique qui entre et sort de la vie de chacun des membres de la famille » (30).

4 C'est à la suggestion du directeur du projet qu'Elliot Liebow choisit donc de s'intéresser à ces hommes noirs «du coin de la rue ", reprenant ainsi une étude pionnière de William Foote Whyte, Street Corner Society, qui, le premier, en 1955, avait fait de ces intersections de rue, où traditionnellement dans un plan en damier s'implantent des commerces, un objet sociologique à part entière. Mais plus que l'ethnographie d'un lieu physique, ce que recherche Liebow est un point d'ancrage où il lui soit donné d'observer l'ensemble des activités sociales des habitants du quartier. Comme le fait remarquer la traductrice dans sa préface, «le street corner sert à la fois de lieu de rencontre, d'échange d'informations, de reconnaissance et d'évaluation, de forum, de refuge, de cadre pour un ensemble d'activités légales ou illégales, publiques ou privées » (15).

5 Liebow a reçu pour toute consigne de trouver un quartier qui puisse être « un bon endroit pour se mouiller les pieds » (142). La ville de Washington, lieu de passage des migrants noirs en provenance du Sud, particulièrement des États de la côte est (Alabama, Géorgie, Caroline du Nord et du Sud, Virginie), a déjà connu une transformation démographique radicale qui a donné aux Noirs la majorité de la population (452000 Noirs sur une population totale de 791000 habitants selon les chiffres de 1962). Liebow connaît bien la capitale et a été témoin de ce processus de succession raciale dans le quartier pauvre où il a lui-même grandi. Né en 1925 de parents juifs, immigrés de l'Europe de l'Est (sa mère était originaire de Lettonie et son 
père de Russie), il avait été témoin du racisme contre les Noirs mais s'était également familiarisé avec eux, qui constituaient une partie de la clientèle de l'épicerie de son père. C'est donc naturellement qu'il choisit de réaliser son enquête de terrain dans un quartier noir de la ville. L'injonction «allez là-bas et comportez vous en anthropologue » (142) le laisse d'abord perplexe, mais très vite, ayant choisi d'explorer un quartier pauvre situé dans le deuxième arrondissement de la capitale, son attention se porte sur un carry out, l'un de ces magasins de vente à emporter qui sert à la fois d'épicerie, de débit de boisson et de restauration rapide. Il abandonne alors son idée initiale de conduire ses recherches dans une succession de terrains et de s'attacher à reconstituer une série de généalogies et d'histoires de vies, et choisit, dans la lignée des grandes monographies de l'École de Chicago, de prendre ce carry out comme unité de lieu où conduire son observation participante. Dans ce décor, les hommes du quartier viennent chercher une "sociabilité sans effort»: "chaque homme vient là principalement parce qu'il connaît ceux qui y seront également. Il vient pour boire et manger, apprécier une discussion facile, apprendre ce qui s'est passé, chahuter, regarder les femmes et badiner avec elles, voir «ce qui se passe » et passer le temps » (39).

6 C'est ainsi que de janvier 1962 à juillet 1963, Liebow vient régulièrement "traîner " dans ce carry out. Il y rencontre et apprend à y connaître un certain nombre d'habitués auxquels il donne des noms fictifs pour protéger leur anonymat, tel le Tally du titre, un homme de trente ans né à Atlanta et arrivé à D. C. en 1954, qui n'a jamais été scolarisé et vit d'expédients, principalement, comme beaucoup de ces hommes noirs, dans le bâtiment où il travaille de manière irrégulière. Liebow précise dans son introduction qu'en huit ans passés à Washington, Tally a eu huit enfants, trois de sa femme et cinq autres avec cinq femmes différentes (39). Pourtant, si des bribes de biographies nous sont ainsi fournies dans l'introduction sur quelques protagonistes de cette étude (Tally, Sea Cat, Richard, Leroy...), ce qui fait l'originalité de Tally's Corner est le refus d'une trame narrative et le choix d'une organisation thématique qui permet en fait de voir ces hommes dans la diversité de leurs rôles sociaux : soutiens de famille (« Des hommes et des emplois ", chapitre 1), pères ("Des pères sans enfants", chapitre 2), maris ("Maris et femmes », chapitre 3), amants (« Amants et exploiteurs », chapitre 4), amis ( Amis et réseaux», chapitre 5), avant de livrer dans une annexe méthodologique ("Retour sur une expérience de terrain»), comme l'avait fait Whyte avant lui dans Street Corner Society, une "profession de foi » (26): Liebow y donne des éléments biographiques et décrit la façon dont il a procédé pour mener à bien son enquête et établir une relation de confiance avec les habitants du quartier.

7 Si Tally's Corner est ainsi devenu une sorte de modèle de l'enquête ethnographique, ce n'est pas tant, comme le souligne Célia Bense Ferreira Alves dans une préface qui met particulièrement bien en valeur les enjeux de ce texte, "par les méthodes de collecte des données utilisées que par les techniques de restitution auxquelles recourt l'auteur » (23). Dans la tradition des monographies des tenants de la tradition de Chicago, Liebow fonde son enquête sur l'observation participante de ce quartier noir en se rendant chaque jour, pendant un an et demi, à ce coin de rue, et en partageant les activités des habitués du carry out, où il lui arrive aussi d'être témoin de certaines scènes ou conversations. Il n'habite pas le quartier, et une fois rentré chez lui, il consigne toutes ses observations. Mais Liebow précise bien dès son introduction qu'il ne prétend pas à la représentativité de la "petite vingtaine d'hommes noirs qui partagent et utilisent, comme point de base de leurs agissements, un coin de rue dans le deuxième 
arrondissement de Washington » (33), pas plus qu'il ne cherche à tester une hypothèse particulière. Il cherche seulement à «donner un sens a posteriori» (33) à ce qu'il lui a été donné d'observer, mais en adoptant ce qu'il appelle "un point de vue interne " (34), c'est à dire en " [organisant] les matériaux autour des relations entre père et enfant, mari et femme, ami et amant" de la façon dont ces hommes eux-mêmes pourraient les organiser (34).

Cette empathie que Liebow a éprouvée avant de la faire partager à ses lecteurs fait de lui un " ethnographe engagé » (27), car le chercheur, sans renoncer à une nécessaire distance critique, veut donner une compréhension affinée du comportement de ces hommes et femmes noirs pauvres, loin des stéréotypes négatifs, véhiculés notamment par le rapport Moynihan, qui vient d'être publié au moment où Liebow rédige son analyse, et qui a suscité une grande polémique sur la stigmatisation de la famille noire, déstructurée et pathogène. Au contraire, Liebow montre, dès son premier chapitre, comment l'exploitation économique et la précarisation dont ces hommes noirs sont victimes de père en fils, parce que la discrimination raciale les confine à des emplois mal rémunérés, non qualifiés et saisonniers, affectent profondément tous les aspects de leur personnalité et conditionnent leur vie, les rendant inaptes à fonder une famille et à entretenir des relations stables avec la mère de leurs enfants. En amenant le lecteur à reconsidérer ses idées préconçues sur le rapport de ces hommes noirs au travail ou à la famille, Liebow semble entretenir "un dialogue permanent avec Daniel Moynihan " (23) et remet en question une à une toutes les représentations que le rapport de ce dernier avait pu contribuer à répandre dans l'opinion, pour montrer comment ces hommes dans l'adversité "survivent et font preuve de résilience» (24), dans leurs relations avec les femmes ou leurs amis. Par dessus tout, ces hommes cherchent à masquer l'échec qu'ils sont voués à connaître dans le travail comme dans leur mariage, en ne pouvant, avec un emploi précaire, subvenir à leurs besoins ni à ceux de leur famille. Liebow conteste aussi la notion de «culture de la pauvreté » pour mettre au contraire l'accent sur ce qu'il y a de commun entre ces hommes et le reste de la société, en dépit des conditions particulièrement dures qu'ils subissent. Dans sa conclusion en particulier, Liebow nie farouchement l'idée d'une «transmission culturelle » de père en fils : « ce qui semble être un processus culturel dynamique et autosuffisant est, tout au moins en partie, une pièce assez simple de la machine sociale qui fabrique, de façon assez mécanique, des sosies produits indépendamment. La question est de savoir comment changer les conditions qui, parce qu'elles garantissent l'échec, font que le fils est fait à l'image du père » (136).

La réponse, pour Liebow, est clairement du côté des pouvoirs publics et des programmes sociaux qu'ils doivent mettre en œuvre pour permettre à ces hommes noirs d'accéder à une bonne éducation puis à un emploi bien rémunéré : «La pauvreté est bien une véritable cible si l'on veut tenter de faire en sorte que les Noirs des classes populaires baignent « dans le cours normal de la vie américaine. [...] Si l'on veut que ce mode de vie change, il faut changer cet élément central. On doit donner à l'homme noir, comme à tout un chacun, les compétences nécessaires pour gagner sa vie et l'occasion de mettre ces compétences à l'œuvre » (136-137).

Plus de quarante après sa première publication, Tally's Corner garde toute sa pertinence et reste aussi un texte singulier qui dresse de manière très détaillée, et finalement rare en sciences sociales, tant aux États-Unis qu'en France, malgré les émeutes urbaines récentes, une chronique de la vie dans les ghettos. Le plus difficile dans cette édition en 
français était de trouver le bon niveau de langue avec cette façon si particulière dont Liebow restitue le discours tenu par ces hommes « du coin de la rue » et insère leurs propos, dans de longues citations, mais aussi parfois au sein d'une même phrase, dans son analyse scientifique. Au-delà des choix de traduction auxquels il a été procédé, la façon dont Célia Bense Ferreira Alves met en lumière tout le contexte politique mais aussi historiographique et épistémologique de cette monographie rend l'enquête de Liebow d'autant plus intéressante que ses thématiques - pauvreté urbaine, précarisation et crise de la masculinité, désorganisation familiale - sont toujours d'actualité.

INDEX

Thèmes : Recensions

\section{AUTEUR}

\section{CATHERINE POUZOULET}

Université Charles de Gaulle- Lille III 